\title{
THE NUMERICAL SPECTRUM OF A ONE-DIMENSIONAL SCHRÖDINGER OPERATOR WITH TWO COMPETING PERIODIC POTENTIALS*
}

\author{
LAURENT GOSSE
}

\begin{abstract}
We are concerned with the numerical study of a simple one-dimensional Schrödinger operator $-\frac{1}{2} \partial_{x x}+\alpha q(x)$ with $\alpha \in \mathbb{R}, q(x)=\cos (x)+\varepsilon \cos (k x), \varepsilon>0$ and $k$ being irrational. This governs the quantum wave function of an independent electron within a crystalline lattice perturbed by some impurities whose dissemination induces long-range order only, which is rendered by means of the quasi-periodic potential $q$. We study numerically what happens for various values of $k$ and $\varepsilon$; it turns out that for $k>1$ and $\varepsilon \ll 1$, that is to say, in case more than one impurity shows up inside an elementary cell of the original lattice, "impurity bands" appear and seem to be $k$-periodic. When $\varepsilon$ grows bigger than one, the opposite case occurs.
\end{abstract}

Key words. quasi-periodic potential, impurity band, quasi-Bloch wave, metallic impurity conduction

\section{AMS subject classifications. $\quad 81 \mathrm{Q} 05,81 \mathrm{Q} 20$}

\section{Introduction and modelling}

This note is devoted to the numerical investigation of the following eigenvalue problem:

$$
\frac{1}{2} \partial_{x x} \psi+(E-\alpha q(x)) \psi=0, \quad q(x)=\cos (x)+\varepsilon \cos (k x) ; \quad x \in \mathbb{R} .
$$

For $\alpha \in \mathbb{R}$ and $\varepsilon=0,(1.1)$ is known as the $2 \pi$-periodic Mathieu (or Hill) equation. Floquet-Bloch theory thus applies to ensure the existence of so-called "Bloch wave solutions" reading $\exp (i \kappa(E) x) z(\kappa, x)$ and indexed by the quasi-momentum $\kappa$ which belongs to the first Brillouin zone. This is also one of the simplest models for the 1-D modelling of an independent electron moving inside a static and infinite lattice of ionic cores; the lows of the potential render the potential wells in the vicinity of the positively charged atomic nuclei. Solving this eigenvalue problem is an important stepping stone toward semiclassical WKB approximation because it allows to apply Peierls' substitution; see $[1,15,12,22]$. For weak and slowly-varying external potentials, this line of thinking still works, including the self-consistent Poisson interaction ${ }^{1}$, because both tunnelling effects and band coupling remain negligible [13, 14].

However, when one wishes to consider defects in the lattice, the resulting perturbations in the potential cease to remain slowly-varying: for instance, a special case of phonon modelling has been studied in [10]. For this model, the band structure has been seen to be deeply modified, but still, consistency between the resulting Schrödinger equation and its semiclassical WKB approximation has been observed numerically. Besides phonons, a situation of particular interest turns out to be the inclusion of impurities inside an otherwise perfectly periodic lattice; in semiconductor

\footnotetext{
${ }^{*}$ Received: January 13, 2007; accepted (in revised version): March 17, 2007. Communicated by Shi Jin.

†IAC-CNR "Mauro Picone" (sezione di Bari) Via Amendola 122/D - 70126 Bari, Italy (l.gosse@ ba.iac.cnr.it).

${ }^{1}$ Hartree equation permits to go beyond the "independent electron" idealization.
} 
theory, this process is called doping [25]. Here we consider (1.1) as the most elementary model for the Hamiltonian of independent electrons permeating a doped material. Weak doping corresponds to low values of $k$, and strong interaction between electrons and nuclei is rendered through a big $\alpha$. The value of $\varepsilon$ may be interpreted as the type of impurity disseminated inside the sample; it somehow controls the strength of the alien ion's effect on the charge carriers.

Unfortunately, the classic Bloch decomposition no longer applies to (1.1), since the equation is only quasi-periodic. Indeed, periodicity is a special case of short-range order, leading generally to an absolutely continuous spectrum (the well-known "band structure") and extended eigenstates; quasi-periodicity, though not being completely disordered, displays only long-range order, thus allowing for singular Cantor-type spectrum and critical (very peaked) eigenstates, some of which can be seen in [11]. What we aim at here are the variations in the spectrum of (1.1) as $k$ and $\varepsilon$ vary; for very low $k$ 's, very few impurities show up in the medium hence based on common belief, the original Mathieu band structure should be visible modulo very small changes, see $\S 3.1$. When $k$ is increased but remains below one, there is less than one impurity inside the Wigner-Seitz cell of the underlying lattice; recent theoretical results (partially recalled in $\S 2$ ) indicate that for $\alpha=O(1)$, one should expect a singular Cantor-type spectrum possibly with gaps, see $\S 3.2$. The opposite situation is of course $k>1$ for which the resulting doping turns out to be very strong; impurity bands then appear inside the band gaps of the unperturbed problem as seen in $§ 3.3$ and in practice correspond to meta-stable states [18, 23].

\section{One-dimensional quasi-periodic Schrödinger operators}

\subsection{A glimpse on theoretical results.}

DEFINITION 2.1. Let $q(x)$ be a smooth function $\mathbb{R} \rightarrow \mathbb{R} ; q$ is said to be quasi-periodic if there exist $d \in \mathbb{N}$ rationally independent constants $\vec{\omega}=\left(\omega_{1}, \ldots, \omega_{d}\right) \in \mathbb{R}^{d}$ and a continuous function $Q: \mathbb{R}^{d} \rightarrow \mathbb{R} 2 \pi$-periodic in each of its arguments such that:

$$
\forall x \in \mathbb{R}, \quad q(x)=Q(\vec{\omega} x) \stackrel{\text { def }}{=} Q\left(\omega_{1} x, \ldots, \omega_{d} x\right) .
$$

The $d$ constants $\vec{\omega}$ are the basic frequencies of $q$, and $Q$ is the (non unique) lift of $q$.

When dealing with quasi-periodic functions, we shall always use capital letters for the corresponding lifts. The study of quantum particles submitted an incommensurate potential started with [22] which was dealing with electrons moving inside a magnetic field. Let $v(x)$ stand for a generic quasi-periodic function, i.e. $v(x)=V(\vec{\omega} x)$ with $\vec{\omega} \in \mathbb{R}^{d}, d \geq 2$. The theory mainly focused on the general eigenvalue problem:

$$
\frac{1}{2} \partial_{x x} \psi(x)+(E-\alpha v(x)) \psi(x)=0, \quad x \in \mathbb{R} .
$$

It states that both eigenfunctions $\psi(x)$ and eigenvalues $E$ strongly depend on $\alpha$. For large $\alpha$, eigenfunctions are known to decay exponentially. This phenomenon is referred to as Anderson localization, see e.g. [8]. But for small $\alpha / E$, the existence of quasi-Bloch waves, that is to say eigenfunctions of the form

$$
\Psi_{\kappa}(x)=\exp (i \kappa(E) x) z(\kappa, x), \quad z(\kappa, x)=Z(\vec{\omega} x),
$$

has been established by means of KAM techniques: consult $[5,21,26,7]$. To get control on the recurrent small divisors, it is necessary to assume that $\vec{\omega}$ is Diophantine, 
i.e. there exist positive constants $C_{0}, \tau$ for which,

$$
\forall \vec{\nu} \neq \overrightarrow{0} \in \mathbb{Z}^{d}, \quad\left|<\vec{\omega}, \vec{\nu}>_{\mathbb{R}^{d}}\right| \geq C_{0}|\vec{\nu}|^{-\tau}, \quad\left|\kappa(E)+<\vec{\omega}, \vec{\nu}>_{\mathbb{R}^{d}}\right| \geq C_{0}|\vec{\nu}|^{-\tau} .
$$

Recently, some interest has been put into perturbed Hill's equations, of which (1.1) is an elementary case; results are available in $[2,9]$. We notice that, besides Diophantine conditions, no particular hypotheses are $\vec{\omega}$; one of the purposes of this paper is to present a case with $d=2$ where the basic frequencies have a critical importance on the structure of the spectrum.

2.2. The incommensurate perturbed Mathieu potential. The behavior of (1.1) in the special case $\varepsilon=1, \alpha \ll 1$ has been studied theoretically in $[8,26]$, numerically in $[4,16,30]$ and experimentally in [28] (see also [27]). From [26, 7, 9], we know that, provided the following Diophantine condition (2.2) holds,

$$
\forall m, n \neq 0 \in \mathbb{Z}^{2}, \quad|m+n k| \geq \frac{C_{0}}{n^{2}},
$$

for $\varepsilon$ small enough and/or $E$ big, "quasi-Bloch waves" $\Psi_{\kappa}(x)=\exp (i \kappa x) z(\kappa, x)$ do exist and the corresponding modulations $z(\kappa,$.$) and q$ share the same kind of quasiperiodicity:

$$
q(x)=\sum_{n, m} \hat{Q}_{n, m} \exp (i(n+m k) x), \quad z(\kappa, x)=\sum_{n, m} \hat{Z}_{n, m}(\kappa) \exp (i(n+m k) x) .
$$

We shall study numerically the case where $k=q \pi$ with $q \in \mathbb{Q}$. This doesn't exactly enter the preceding framework as, despite being irrational, $\pi$ isn't an algebraic number. Thus it isn't known if the corresponding frequencies can be Diophantine. The best which seems to have been proven ${ }^{2}$ is: $\left|\pi-\frac{p}{q}\right| \geq q^{-42}$. For completeness, we recall the continued fraction approximation of $\pi$ together with its graphical representation on Fig.2.1:

$$
\begin{array}{r}
\pi \simeq[3,7,15,1,292,1,1,1,2,1,3,1,14,2,1,1,2,2,2,2,1,84,2,1,1,15,3,13,1,4,2,6,6, \\
99,1,2,2,6,3,5,1,1,6,8,1,7,1,2,3,7,1,2,1,1,12,1,1,1,3,1,1,8,1,1,2,1,6,1,1, \\
5,2,2,3,1,2,4,4,16,1,161,45,1,22,1,2,2,1,4,1,2,24,1,2,1,3,1,2,1] .
\end{array}
$$

However, it seems also interesting to check numerically whether or not the results based on Diophantine frequencies can extend to irrational numbers possibly better approximated by rational numbers. In order to study numerically the slightly more general problem (1.1) with respect to $\alpha, k$ and $\varepsilon$, it is easy to extend the spectral algorithm proposed by Steve Surace in [26]:

$$
(n+m k+\kappa)^{2} \hat{Z}_{n, m}+\alpha\left(\hat{Z}_{n-1, m}+\hat{Z}_{n+1, m}+\varepsilon \hat{Z}_{n, m-1}+\varepsilon \hat{Z}_{n, m+1}\right)=2 E_{\kappa} \hat{Z}_{n, m} .
$$

Due to CPU time, we restricted our computations to $n, m \in\{-20,20\}$, which lead to diagonalizing a $41^{2} \times 41^{2}$ penta-diagonal matrix for every value of $\kappa$. We always took $\alpha=1$.

\section{Numerical results and discussion}

Hereafter, we shall always consider Figure 1 in [12] as the reference for the band structure of (1.1) with $\alpha=1$ and $\varepsilon=0$, i.e. of the classical Mathieu equation. We recall that in this case, bands are to be visualized for $\kappa \in\left[-\frac{1}{2}, \frac{1}{2}\right]$, the first Brillouin zone.

\footnotetext{
${ }^{2}$ see http://mathforum.org/library/drmath/view/69162.html
} 


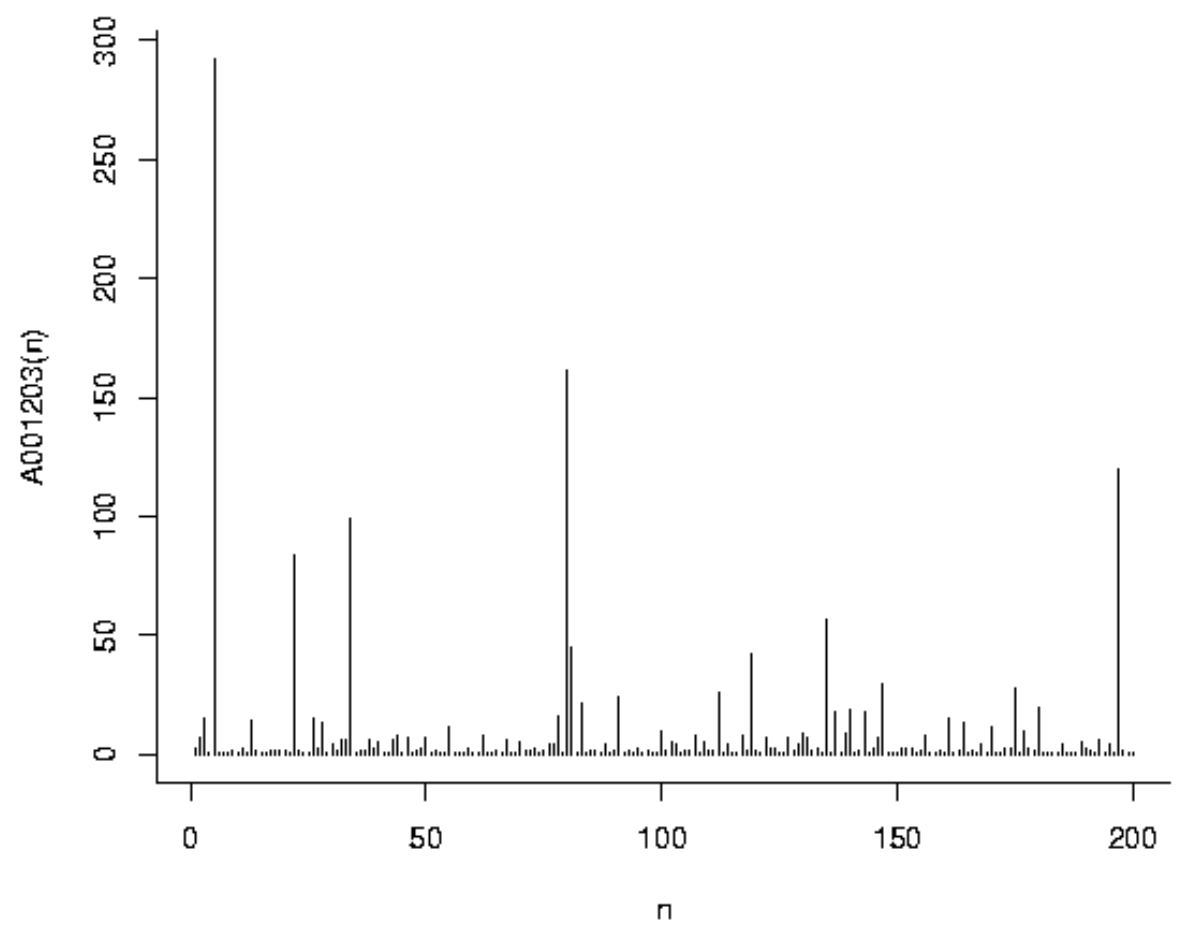

FIG. 2.1. Graphical representation of the continued fraction approximation of $\pi$.

3.1. Increasing $k$ : from adiabatic decoupling to disorder. In this case, we took $\varepsilon=0.15$ and let $k$ vary while remaining inside the interval $[1 / 300,1 / 10]$. The numerical results are displayed on Fig. 3.1 for $\kappa$ inside the Brillouin zone of the original problem. One sees at once that the lower $k$ is, the more the spectrum looks like the Mathieu's bands. Indeed, states located deep down the well are nearly flat and a conduction band is visible near the the edge of the potential. We believe that the "thickness" of the bands has to do with the degeneracy of the (big) matrix coming from (2.4) as many eigenvalues are very close to each other and this may influence the numerical diagonalization algorithm; the higher $\varepsilon$, the thicker these bands.

However, if the structure is almost perfect for $k \simeq 1 / 300$, it surely evolves toward a different picture as $k$ increases. Indeed, the bands already begin to flatten for $k \simeq 1 / 100$, meaning that localized states begin to be numerous. Finally, for $k \simeq 0.1$, nearly all the bands have become flat, except for the conduction band, which displays an intriguing structure. Fig. 3.1 (especially the left spectrum) tells us that if the perturbation potential is really slowly-varying, then Peierls substitution is completely effective and the electron's dynamics are driven by usual energy bands (as studied in [13]). Band gaps are still present in the spectrum for all the considered values.

3.2. Weak disorder: Cantor spectrum for various values of $\varepsilon$. Here we took $k=\frac{\sqrt{5}-1}{2} \simeq 0.62$, the golden mean, and we want to investigate the stability 

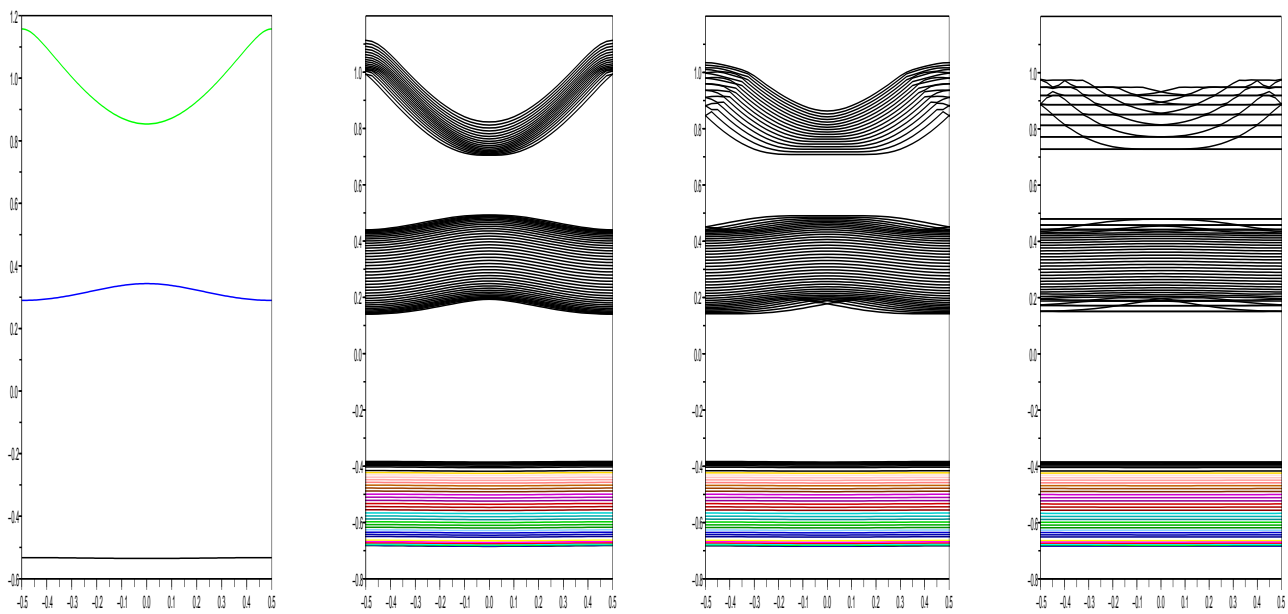

FIG. 3.1. Spectrum for $\varepsilon=0.15$ with $k=\frac{\pi}{1000}, \frac{\pi}{250}, \frac{\pi}{50}$ (left to right). Lowest homogeneous Mathieu-Hill's $(\varepsilon=0)$ bands are shown on the left for comparison.

of the band gaps as $\varepsilon$ varies. First of all, we see that all the original bands have collapsed into a complicated "Cantor-type structure" as a consequence of the band nesting phenomenon, see [16], to which critical eigenstates ${ }^{3}$ should be associated. Then, band gaps are stable; they roughly agree with former computations for the simpler model $\varepsilon=1, \alpha \ll 1$ given in [30]. Indeed, the increase of $\varepsilon$ only thickens the "Cantor areas" and restricts a bit these forbidden zones; this is reminiscent of the Peierls distortion in which phonons open small gaps inside the conduction band. It looks also like a smooth process as has been shown in [2] using the concepts of "resonance tongues" and "instability pockets". The upper part of the spectrum is rather messy, so we didn't display it; see however [11].

3.3. Strong disorder: $k$-periodic impurity bands for $k>1$. Here, we focus on the case for which $k=\frac{\pi}{3}>1$ and let $\varepsilon$ vary. Gaps completely disappeared at the benefit of new "impurity bands"; physically, this is a consequence of the excessive overlapping of the wave functions associated to the impurity atoms. In the opposite cases corresponding to Figs. 3.1 and 3.2, impurity wave functions were meant to stay far enough from each other to prevent band creation. In case $k>1$, however, they became closer than the states created by the original Mathieu potential. Moreover, they are quite stable as can be seen on Fig. 3.3, despite the "thickness" of the collapsed bands still growing with $\varepsilon$. The new bands look like being $k$-periodic hence the original Brillouin zone is now too narrow to include them; see also [11] for a similar observation with a different type of perturbation. In [11], we observed that impurity bands appear to be parabolic: a plausible interpretation is that electrons

\footnotetext{
${ }^{3}$ Extended states are associated to periodic problems $(\varepsilon=0)$. Localized states are usually associated to random operators; they have a finite $L^{2}(\mathbb{R})$ norm and decay exponentially for large $|x|$, see $[4,8]$. Critical states are in between as they are usually made of periodically-repeated spikes; see [17] and $\S 4$ in [11] for an illustration.
} 

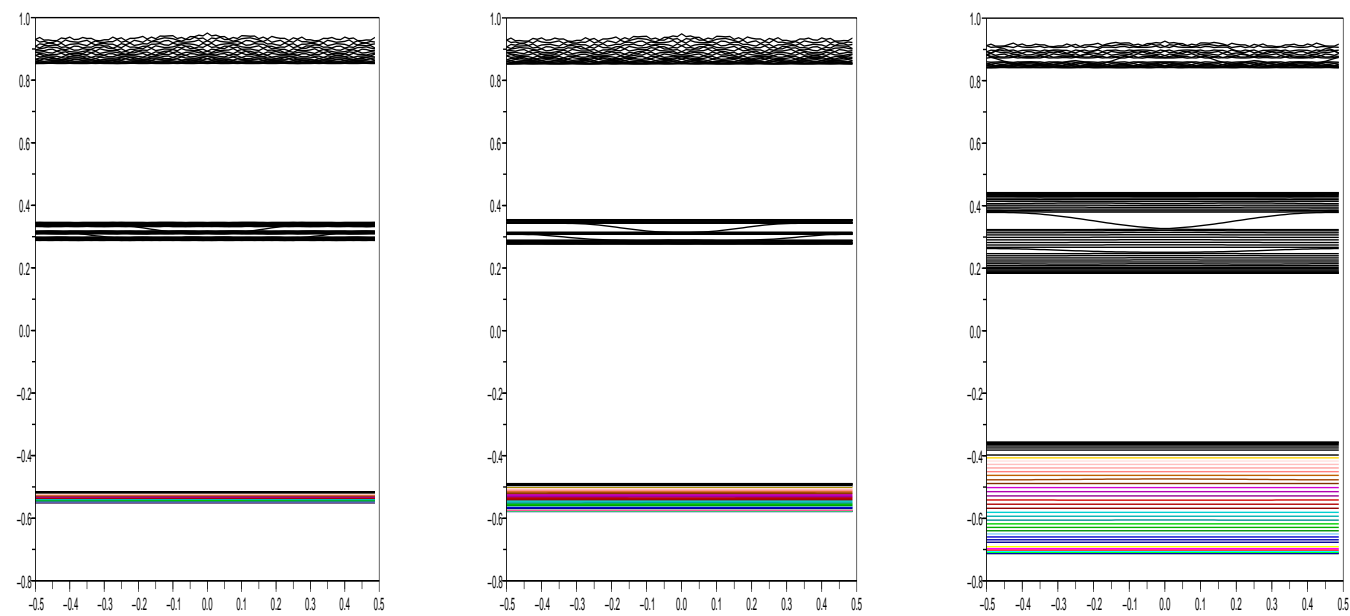

FIG. 3.2. Spectrum with $k=\frac{\sqrt{5}-1}{2}$ and $\varepsilon=0.02,0.05,0.2$ (left to right).
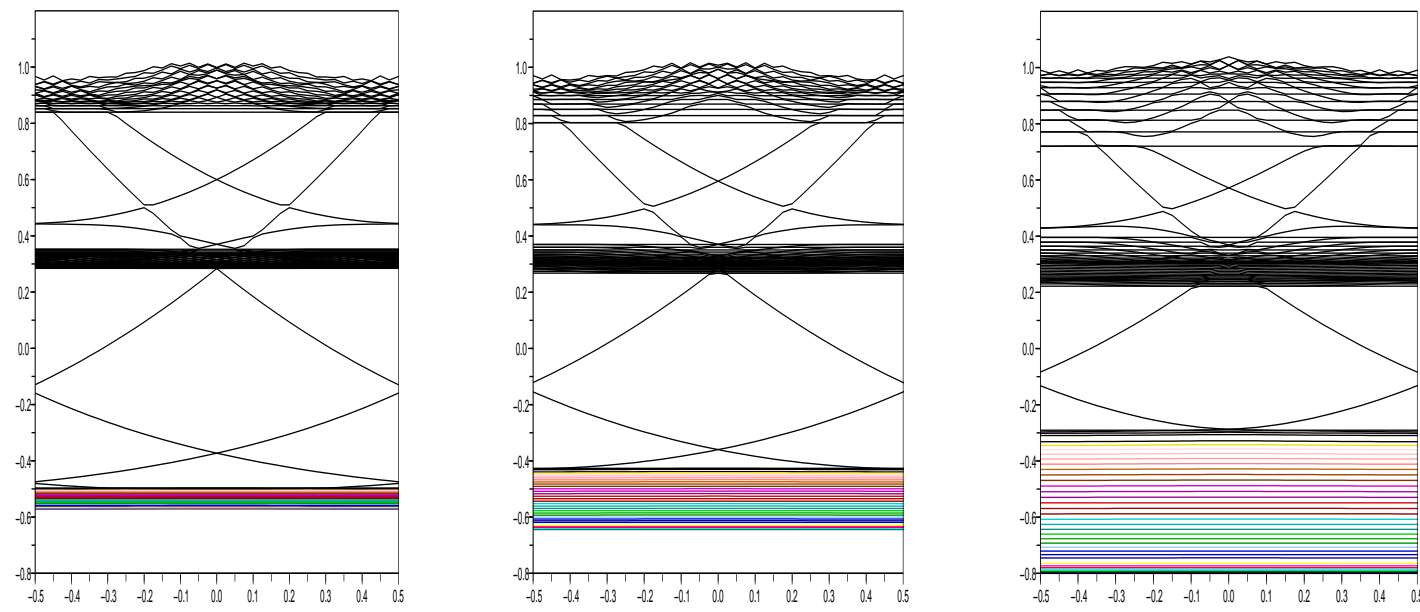

FIG. 3.3. Spectrum with $k=\frac{\pi}{3}$ and $\varepsilon=0.05,0.15,0.35$ (left to right).

perceive somehow the average of the corresponding very oscillating potential (like the so-called Jellium metal model [6]) and thus display a parabolic dispersion relation. The effect of the disordered medium boils down to the effective mass only.

3.4. What happens if $\varepsilon>1$ ? One may imagine that for $\varepsilon$ big enough, the collapsed bands will become so thick that no space will be left for the impurity bands and the resulting sample will be an insulator. This is only partly true, in the sense 

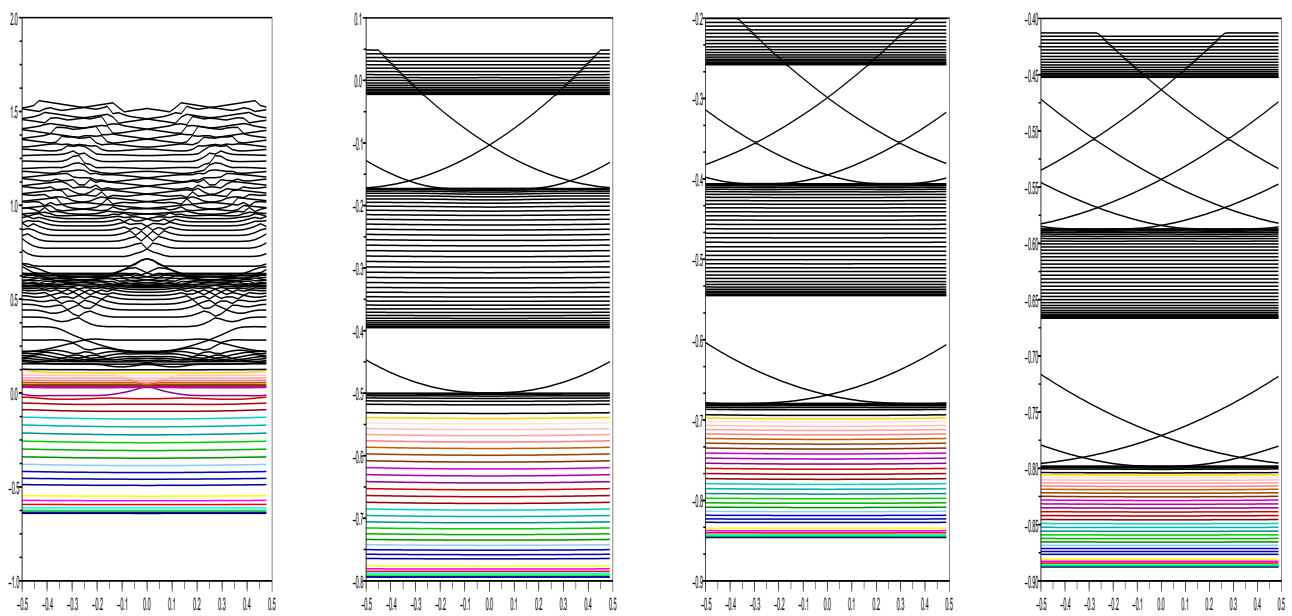

FIG. 3.4. Spectrum with $k=\frac{\pi}{3}$ and $\varepsilon=0.85,5,10,20$ (left to right): only localized states separated by gaps are expected to survive.
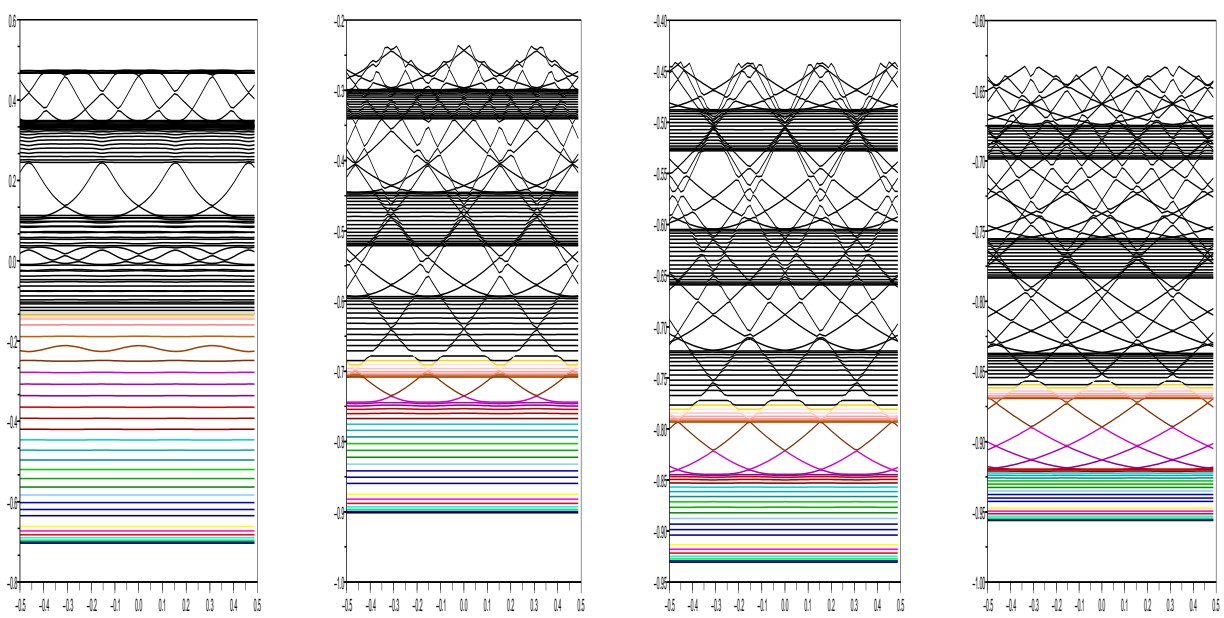

FIG. 3.5. Spectrum with $k=\frac{\sqrt{5}-1}{2}$ and $\varepsilon=0.85,10,20,50$ (left to right). Extended states can be expected to develop as $\varepsilon$ grows.

that in case $\varepsilon$ becomes greater than one, the "perturbation" $\varepsilon \cos (k x)$ becomes the main potential and thus the term $\cos (x)$ reduces to a lower-frequency perturbation. One can apply the transformation $x \rightarrow k x$ inside problem (1.1) with $\alpha=1$ in order to 
derive:

$$
\frac{k^{2}}{2} \partial_{x x} \psi+(E-\varepsilon \cos (x)-\cos (x / k)) \psi=0 ; \quad x \in \mathbb{R}
$$

For values of $k$ close to one, the factor in front of the second derivative won't be important; however, one sees at once that in case $\varepsilon>1$, impurity bands should appear in the opposite case, that is to say for $k<1$; this is reminiscent of the mobility edge phenomenon. So the picture goes as follows: singular spectrum grows in size for $0.2 \leq \varepsilon<1$, then for $\varepsilon>1$, we gradually move back to the situation described in Fig. 3.2 or 3.3 ; we display on Fig. 3.4 the spectrum resulting from "big" values of $\varepsilon$, but in order to ease the comparison, we divided all eigenvalues by $1+\varepsilon$. The thickness of the singular parts decreases slowly and we believe that the bands remaining inside the gaps result from truncation errors; observe in particular the similarity between the spectrum on the right of Fig. 3.1 and the one on the left of Fig. 3.4. For completeness, we display on Fig. 3.5 what happens for increasing values of $\varepsilon$ in case $k=\frac{\sqrt{5}-1}{2}$; the results suggest that some bands emerge between the singular parts of the spectrum which size gradually shrinks (like in the preceding Fig. 3.4). For $\varepsilon<1$, we observe other bands high in the spectrum; this isn't in contradiction with our ideas since standard KAM results (see especially [7, 9]) ensure that higher parts of the spectrum generally contain extended states. Notice also that in this case, the "perturbing potential" is still endowed with a Diophantine frequency since $\frac{1}{k}=\frac{\sqrt{5}+1}{2}$, the inverse of the golden mean, has a continued fraction representation going like $1+[1,1,1,1 \ldots]$.

\section{Conclusion}

We presented some computational results obtained from a simple spectral algorithm (2.4), which nevertheless has been studied rigorously in [26] in a related context. We observed that for very dilute perturbations, the original band structure survives, but it quickly rearranges itself as the parameter $k$ grows. Metallic conduction can be hoped for, but it should decay with $k$. For $k$ slightly below one, that is, the frequency of the underlying lattice, the spectrum is singular continuous, and thus eigenstates are likely to be critical. The resulting material will therefore be an insulator. Amazingly, for $k>1$, intricate "impurity bands" show up inside an otherwise singular spectrum; hence assuming that electrons might be trapped in these bands, frictionless conduction remains possible. It seems to be a well-known result that certain forms of disorder lead to the formation of impurity bands responsible for metallic impurity conduction in the sense of [20], see also [3, 25, 29]. This is also used for the design of semiconductor LASERs, where the stimulated emission of radiation stems from an inverted population of electrons decaying from an excited band onto a meta-stable one.

Acknowledgements. This work was partially supported by o Centro de Matemática e Aplicações Fundamentais (Lisbon), funded by FCT as contract POCTIISFL-1-209, and by the Austrian Ministry of Science via its grant for the Wolfgang Pauli Institute and by the Austrian Science Foundation (FWF) via the START Project (Y-137-TEC).

\section{REFERENCES}

[1] N. W. Ashcroft and N. D. Mermin, Solid-State Physics, Holt; Rinehart and Winston, 1976. 
[2] H. Broer, J. Puig and C. Simó, Resonances tongues and instability pockets in the quasi-periodic Hill-Schrödinger equation, Comm. Math. Phys., 241, 467-503, 2003.

[3] C. di Castro and R. Raimondi, Disordered electron systems, arXiv: cond-mat/0402203, 5, preprint.

[4] R. B. Diener, G. A. Georgakis, J. Zhong, M. Raizen and Q. Niu, Transition between extended and localized states in a one-dimensional incommensurate optical lattice, Phys. Rev. A, 64, 033416, 2001.

[5] E. Dinaburg and Yakov Sinaï, The one-dimensional Schrödinger equation with quasi-periodic potential, Funkt. Anal. i Priloz., 9, 8-21, 1975.

[6] W. Eckardt, Dynamical polarizability of small metal particles: self-consistent spherical jellium background model, Phys. Rev. Lett., 52, 1925, 1984.

[7] L. H. Eliasson, Floquet solutions for the 1-dimensional quasi-periodic Schrödinger equation, Comm. Math. Phys., 146, 447-482, 1992.

[8] J. Fröhlich, J. Pöschel and P. Wittawer, Localization for a class of one-dimensional quasiperiodic Schrödinger operators, Comm. Math. Phys., 132, 5-25, 1990.

[9] G. Gentile, D. A. Cortez and J. C. A. Barata, Stability for the quasi-periodically perturbed Hill's equation, Comm. Math. Phys., 260, 403-443, 2005.

[10] L. Gosse, Multiphase semiclassical approximation of the one-dimensional harmonic crystal. I. The periodic case, J. Phys. A Math. Gen., 39, 10509-10521, 2006.

[11] L. Gosse, Impurity bands and quasi-Bloch waves for a one-dimensional model of modulated crystal, Nonlinear Analysis, to appear, 2006.

[12] L. Gosse and P. A. Markowich, Multiphase semiclassical approximation of an electron in a one-dimensional periodic lattice. I. Homogeneous problems, J. Comp. Phys., 197, 387-417, 2004 .

[13] L. Gosse, Multiphase semiclassical approximation of an electron in a one-dimensional crystalline lattice - II. Impurities, confinement and Bloch oscillations., J. Comp. Phys., 201, 344-375, 2004.

[14] L. Gosse and N. J. Mauser, Multiphase semiclassical approximation of an electron in a onedimensional crystalline lattice - III. from ab-initio models to WKB for SchrödingerPoisson, J. Comp. Phys., 211, 326-346, 2006.

[15] J. C. Guillot, J. Ralston and E. Trubowitz, Semiclassical asymptotics in solid-state physics, Comm. Math. Phys., 116, 401-415, 1988.

[16] J. M. Hollingworth, A. Vourdas and N. Backhouse, Wave propagation in one-dimensional optical quasiperiodic systems, Phys. Rev. E, 64, 036611, 2001.

[17] M. Kohmoto, B. Sutherland and C. Tang, Critical wave functions and Cantor-set spectrum of a one-dimensional quasicrystal model, Phys. Rev. B, 35, 1020-1033, 1987.

[18] Melvin Lax and James C. Phillips, One-dimensional impurity bands, Phys. Rev., 110, 41-49, 1958.

[19] W. Man, M. Megens, P. J. Steinhart and P. M. Chaikin, Experimental measurement of the photonic properties of icosahedral quasicrystals, Nature, 436(18), 993-997, 2005.

[20] N. F. Mott and W. D. Twose, The theory of impurity conduction [Part II], Advances in Physics, 10(38), 107-163, 1961.

[21] J. Moser and J. Pöschel, An extension of a result by Dinaburg and Sinai on quasi-periodic potentials, Comment. Math. Helvetici, 59, 39-85, 1984.

[22] R. E. Peierls, Quantum Theory of Solids, Clarendon, Oxford, 1955.

[23] J. J. Rehr and W. Kohn, Impurity states between two bands, Phys. Rev. B, 9, 1981-1983, 1974.

[24] E. Rotenberg, W. Theis, K. Horn and P. Gille, Quasicrystalline valence bands in decagonal Al-Ni-Co, Letters to Nature, 406(10), 602-605, 2000.

[25] B. Shklovskii and A. L. Effros, Electronic Properties of Doped Semiconductors, Springer Verlag, 1984.

[26] S. Surace Jr., The Schrödinger equation with a quasi-periodic potential, Trans. Amer. Math. Soc., 320, 321-370, 1990.

[27] M. A. Torres, J. P. Adrados, J. L. Aragon, P. Cobo and S. Tehuacanero, Quasi-periodic Blochlike states in surface wave experiment, Phys. Rev. Lett., 90, 114501, 2003.

[28] J. Voit, L. Perfetti, F. Zwick, H. Berger, G. Margaritondo, G. Grüner, H. Höchst and M. Grioni, Electronic structure of solids with competing periodic potentials, Science, 290, 501503,2000

[29] C. T. White and T. N. Todorov, Carbon nanotubes as long ballistic conductors, Letters to Nature, 393, 240-242, 1998.

[30] R. S. Zounes and R. H. Rand, Transition curves for the quasi-periodic Mathieu equation, SIAM J. Appl. Math., 58, 1094-1115, 1998. 